\title{
Reconfigurable Self-optimising Handling System
}

\author{
Rainer Müller ${ }^{1}$, Martin Riedel ${ }^{2}$, Matthias Vette $^{1}$, Burkhard Corves ${ }^{2}$, \\ Martin Esser ${ }^{1}$, and Mathias Hüsing ${ }^{2}$ \\ ${ }^{1}$ Laboratory for Machine Tools and Production Engineering (WZL), \\ Chair of Assembly Systems, RWTH Aachen University \\ ${ }^{2}$ Department of Mechanism Theory and Dynamics of Machines (IGM), \\ RWTH Aachen University \\ $\{$ R.Mueller,M.Esser, M.Vette\}@wzl.rwth-aachen. de \\ \{Riedel, Corves, Huesing\} @igm.rwth-aachen. de
}

\begin{abstract}
Demand for more versatile assembly and handling systems to facilitate customised production is gaining in importance. A new handling principle has been developed as a cost-effective approach to adapt to component-dependent tasks. It is based upon the gripping and movement of objects by multiple arms within a parallel kinematic structure. This structure combines the advantages of a system of co-operating robots with a simplified drive concept, in which the number of drives used is sharply reduced. On this basis, a modular assembly platform is being developed which, in addition to the kinematic units, also facilitates the integration of measurement, testing and joining modules. The modular concept also creates the conditions for a versatile, demand-driven layout of multiple kinematic units. This facilitates not only cooperative handling of large components using several gripping points, but also the transfer of objects handled between the individual units. These features of adaptivity are the basis for self-optimisation, which then can be implemented within a suitable control system.
\end{abstract}

\section{Introduction}

There has been a fundamental change in the conditions governing manufacturing industry in recent years. Progressive globalisation, rapid technological development and changes in the resources situation [1], [2] are responsible for increasing complexity and dynamics in industry and the industrial environment. One of the consequences is a further reduction of product life cycles, a sustained increase in the number of versions of products and constant pressure to cut manufacturing costs [3]. Assembly systems and processes are particularly exposed to these pressures, as they add a large part of the value in the manufacturing process.

\section{Motivation}

Progressive development in the field of industrial robotics has led to a variety of new applications in recent years, safeguarding jobs in high-wage European states by increasing the level of automation. 
Traditional demands on handling systems are currently undergoing change. In the past, higher and higher load capacity, greater and greater precision and higher and higher speeds have been demanded. However, priorities are increasingly shifting towards customised production and flexible solutions to component-dependent problems. Currently available handling systems frequently cannot fulfill the increased demands and complex tasks [4].

Against this background, one important current problem is the handling of large components, some of which have no intrinsic rigidity. Such components are used in aerospace systems, shipbuilding, wind turbine construction and the manufacture of solar panels. The trend is predominantly discernable in aircraft construction, where larger and larger shell elements in carbon-fibre reinforced plastics are being used for aircraft fuselages [5]. In the particular case of carbon-fibre reinforced plastics, care must be taken not to subject the components to any high forces during handling. Great precision and a large workspace are required. A single robot is not usually capable of moving the component without subjecting it to any forces. Large jigs are therefore used, rendering the system very inflexible and expensive.

Co-operating robots represent a more versatile approach to the problem described. This concept enables inflexible shapes to be dispensed with, as the component can be gripped and supported at different points by several robots [6]. The high procurement costs of industrial robots must be mentioned as a disadvantage. Complicated programming, which entails considerable set-up times, frequently means that such robot systems cannot be used cost-effectively.

Parallel kinematic structures are a cost-effective alternative. Even large, heavy components can be positioned quickly and accurately by closed kinematic chains. A disadvantage is the small workspace dictated by design, which is why parallel kinematic systems frequently do not represent an ideal solution to a general handling problem [7]. Therefore, a handling concept, which combines serial and parallel approaches, was designed.

\section{Assembly Concept}

An assembly platform which can be reconfigured for a specific task is required, particularly for large components in small series and with a lot of versions. The usual limits, such as the workspace and the range of components and use, have to be overcome to create an adaptable system which is reconfigurable and universally useable. A novel handling system has been developed. It is based upon gripping and moving objects by the use of multiple arms within a parallel kinematic structure. The parallel kinematic structure is regenerated with each gripping movement and integrates the object within the robot structure as a moveable platform, as shown in Fig. 1 [8], [9], [10], [11].

This approach combines the handling technology in known robot solutions to form a new overall solution, which is superior to the existing individual solutions in terms of reconfigurability, adaptability, cost-effectiveness and efficiency.

The main concept is based on the motion of a parallel robot, see Fig. 2. In this case, the object is bound to a moveable platform by a gripper, which is moved through the workspace by several kinematic chains. The joints on the platform combine the individual arms to form closed kinematic chains. 


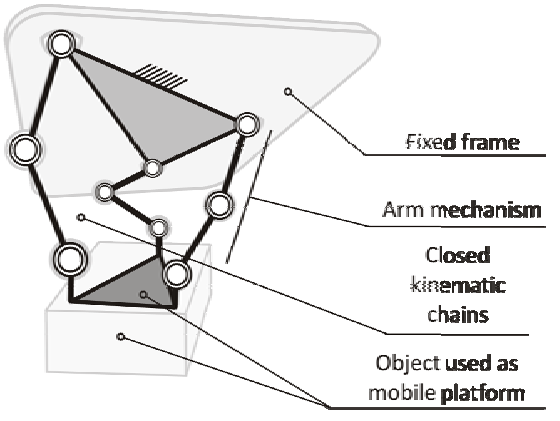

Fig. 1. New handling principle

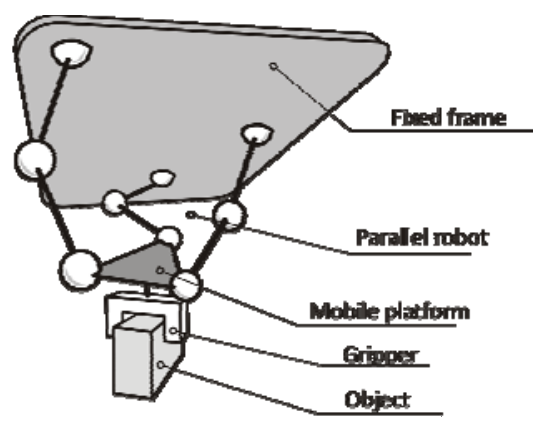

Fig 2. Handling with a parallel robot

The arrangement and type of platform joints are essential to the kinematic structure, whilst the shape and size of the platform have no influence on the kinematics. If the joints are placed directly on the object handled, the object can be integrated into the structure as a platform and be moved by the system. The newly generated kinematic structure is similar to the structure of parallel robots. In contrast to cooperating robots, the configuration of the architecture in each object pose is determined by only six drives.

The handing method derived from this concept distinguishes three phases of movement, gripping, manipulating and depositing, shown in Fig. 3.

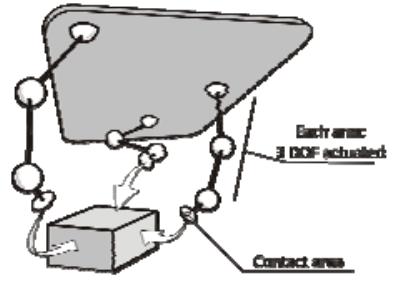

Phase 1: gripping

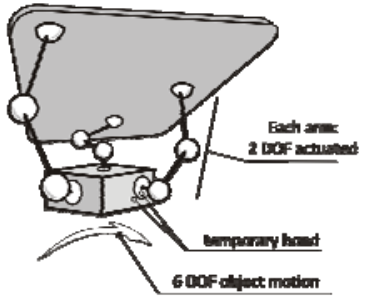

Phase 2: manipulation

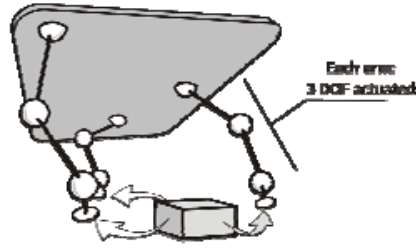

Phase 3: releasing

Fig. 3. Movement sequence when handling an object

In the first phase (gripping), the object is free and the arms can move independently from each other to locate the contact elements on the object. These desired contact points are the later joint positions on the mobile platform. Three drives per arm are necessary to control the 3 degrees of freedom (DOF) of the individual arm. The contact elements are mounted swivelling and spring-centred in the wrist joints with additional 3DOF of rotation. At contact they align passively with the surfaces of the object.

In the second phase (manipulation), the contact elements create a bond with the object which can transmit all directions of forces and moments. The bond may be based upon various physical principles, e.g. form closure, temporary adhesion, subatmospheric pressure, magnetism, etc. The firm but pivoting bonds integrate the object into the structure and can now be moved as the latter's platform with $6 \mathrm{DOF}$. As the 
newly-formed structure is determined, only six drives are required for spatial object movement. Active positioning of the contact elements on the object establishes the characteristics of the structure, such as workspace, payload, transmission of velocity, accuracy, stiffness, etc, by means of the kinematic dimensions. If given appropriate consideration, these can be combined individually and optimally for the respective handling task with every grip, thus creating the basis for self-optimization.

In the third and final movement phase (releasing), the object is released, the contact forces are relieved and the arms are moved away, independently of each other.

In the second phase (manipulation), only two controlled drives are necessary for each arm, whilst the other two phases require an additional drive per arm. This additional drive serves only to position the contact elements during gripping and depositing and, as an auxiliary drive, can be designed as less powerful than the total of six principal drives which move the object. The secondary drives can be driven passively or decoupled in phase two. However, the drives can also provide active support to object movement if required.

Contemporary robotic solutions in which multiple integrated six-axis industrial robots move a component provide comparable payload capacity and versatility in use. Nevertheless, there are fundamental differences in drive configuration. The six drives in the new configuration contrast with the eighteen drives of a conventional "threerobot cell", entailing a considerable cost advantage for the new solution with comparable functionality.

The broad spectrum of use and adaptability of the new handling unit to changing tasks is achieved by two-stage convertibility of the system, Fig. 4.

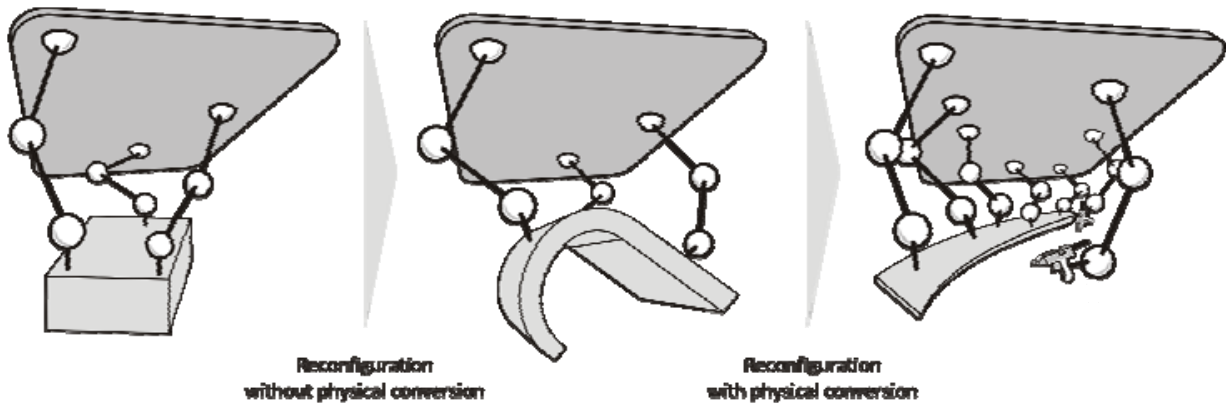

Fig. 4. Two-stage convertibility of the handling system

The first stage is adjustment of the kinematic dimensions of the platform to the imminent object movement. This adjustment can take place automatically on integration of the object into the structure by gripping. It does not require any physical conversion of the unit frame. This is a first step towards the goal of self-optimizing handling.

If the changes in the characteristics and dimensions of the objects handled are too great or if further functions such as measurement, testing or machining are to be integrated into the movement sequence, the system can be adjusted by conversion in a second stage. The base joints of the arms are mounted on a system of rails so that they can be moved easily. Additional kinematic units or modules can be integrated simply and quickly in a modular system. 


\section{Development Process}

The development of such a handling system can traditionally be divided into analysis of requirements, concept, structural synthesis, dimensional synthesis, layout of secondary dimensions and design phases. The results of the concept phase have already been summarised in the previous paragraph. The subsequent developmental stages are described briefly below.

In an early stage of development, the kinematic structure is established so that the desired object movement is possible with $6 \mathrm{DOF}$.

Adapting Grübler's criteria demonstrates that the connectivity number of each arm must be equal to 6 to move objects freely within the workspace, regardless of the number of arms.

To identify suitable arm structures, it is expedient to break down the overall $6 \mathrm{DOF}$ for each arm into a regional structure (3 DOF for translation) and local structure (3 DOF for rotation) and to look for partial solutions independently of each other. This procedure precludes structures with a purely parallel kinematic arm structure from the outset. These solutions would reduce the workspace of the arm assembly significantly.

A systematic approach facilitates full inclusion of the solutions for the partial structures with 3 DOF in translation. Both serial and parallel kinematic chains as well as combinations of both (hybrid structures) are considered. The comprehensive host of solutions is reduced to a small number of possible structures by restrictions in a preselection process. They are analysed individually and assessed using the criteria in the process compliant with VDI 2225.

The finally selected kinematic structure is shown in Fig. 5.

A detailed description of the development process and the kinematic computation of the structure are given in [8], [9], [11] and [12].

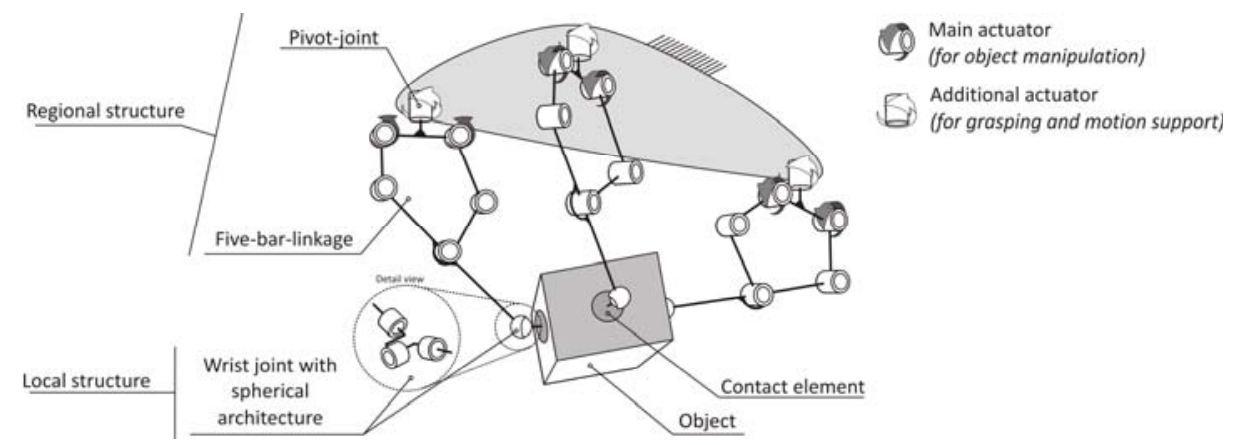

Fig. 5. Selected kinematic structure

After all the principal parameters were determined, the other components were projected and designed in detail on a CAD system. Questions such as component layout, types of connection and suitability for fabrication and installation are considered, producing the prototype design shown in Fig. 6. 
The prototype consists of three arms pivot-mounted on the fixed frame so that they can rotate vertically and articulated to the object during the latter's movement. The fixed frame measures approximately $1300 \times 1500 \mathrm{~mm}^{2}$ (W x D) and allows the arms to be applied in different configurations.

Each arm is $900 \mathrm{~mm}$ long in its extended state and consists of a five-bar linkage parallelogram as the guidance mechanism with a tilting frame. This tilting frame carries both the servomotors which drive both cranks of the parallelogram by belts. The drives are located so that their inertia effect is minimal in an acceleration of rotation of the arm about the vertical axis. The vertical axis is also driven by a servomotor/belt combination, the size of which can be reduced by the mitigated demands of the gripping function. The five-bar linkage moves the wrist joint with the contact element. In initial prototypes, an electromagnet with a weight of $300 \mathrm{~g}$ is used, capable or generating a temporary bonding force of $700 \mathrm{~N}$. A gas-pressurised spring relieves the drives when lifting heavy components, thus increasing the load-bearing capacity.

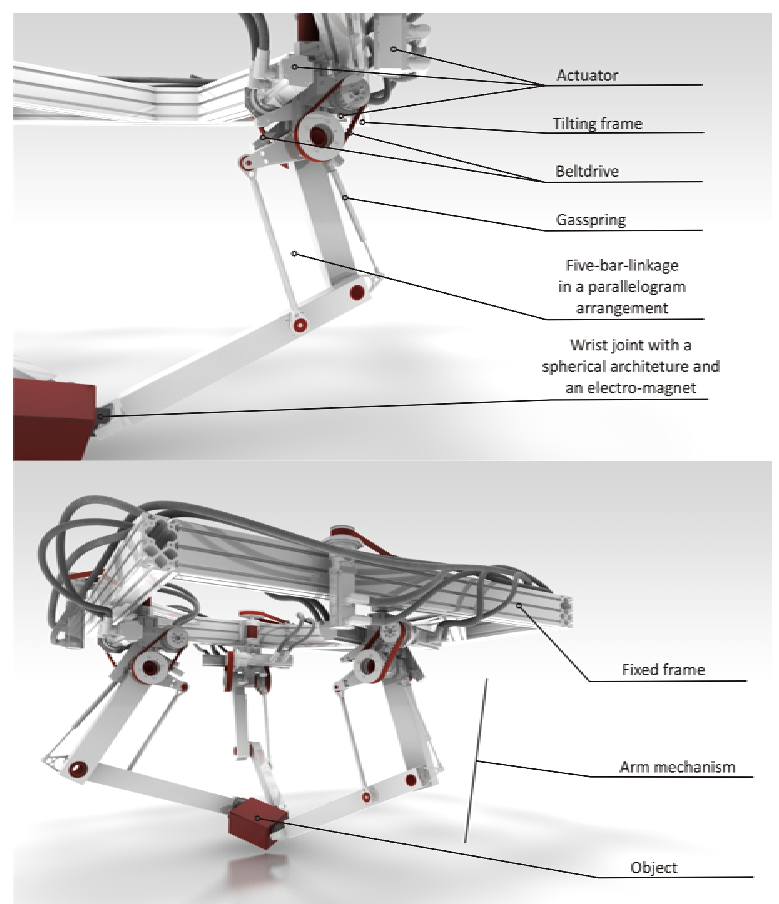

Fig. 6. CAD model of the prototype design

\section{Application}

The objective is the production of a platform concept which can be configured to the task. Beside the kinematic units, further modules are to be integrated. They include measurement and testing modules, and modules for joining and assembling components. 
Additional units and drives can be used to statically over-determinate the system and keep the work piece free of kinematic load.

One specimen application of such an assembly platform is stringer installation in aircraft construction. In this case challenges arise predominantly from the spherical curvature of the shell components and the accompanying stringers of different sizes and curvature which have to be handled. A further difficulty is that the stringers, which are up to $15 \mathrm{~m}$ long, are limp and therefore have to be supported by additional grippers. Modularisation enables the kinematic units to be positioned suitably.

Cooperative handling produces a further advantage. A stringer can be passed on to another kinematic unit before it leaves the workspace of a kinematic unit. Similar concepts are also used in cooperating robots, in which parts are passed from one robot to another. Large workspace can then be covered by relatively small handling units. Such transfer of components cannot usually be achieved cost-effectively by conventional industrial robots, due to the high number of kinematic units required.

\section{Self-optimisation}

The degrees of freedom of the introduced system offer adaptivity for a wide spectrum of handling tasks. Nevertheless, they also imply a large planning effort for finding and optimizing the configuration needed for an individual handling task. Thus the benefit of adaptivity is reduced. Self-Optimizing, i.e. automatically generated configuration for the best possible handling solution is necessary for the cost-effective use of the assembly platform's reconfigurability.

The information needed to perform automatic optimization are the handling task (shape and path of the part) and the configuration of the handling system.

The user will have the opportunity to specify the path in a virtual 3D-model and simulate the process after automatic calculation of the optimal configuration. Reconfiguration of the moving platform is then performed automatically by re-gripping the part at the proposed locations. Reconfiguration of the number and base points of kinematic units will be done manually. After this, the precise kinematic parameters for the assembly platform can be identified automatically using an external measuring system, e.g. iGPS. This has the advantage of reducing set-up time further as the kinematic units do not have to be aligned precisely by hand.

The handling motions are then calculated by using the real geometric parameters of the system and the given path of the work piece. Additional manual adjustment of the path, as in conventional off-line programming, can largely be waived.

\section{Summary and Outlook}

A reconfigurable handling concept has been introduced that combines the advantages of cooperating serial robots and parallel kinematics to overcome the use of large fixtures when handling small series of large parts. The design and application of the concept has been shown and it was made clear, that the reconfigurability can offer significant economical advantages, when the increased planning effort is addressed by means of selfoptimization. Based on the mechanical and control development described within this paper, the chosen concept of self-optimization will be further developed and implemented. 
Interface management constitutes a further important aspect. To facilitate development of an adaptable assembly platform, it must be ensured that other objects can also be integrated, as well as the developed kinematic units. Further handling, control, measurement and test systems are conceivable. For this reason a modular open control system has been selected for future expansion of the system.

The developed kinematic units, existing conventional robotic systems, measurement systems, etc could be integrated to meet demand practically without restrictions and used in a single system against a background of consistent mechatronic modularisation, on the basis of the common platform.

The depicted research has been funded by the German Research Foundation DFG as part of the Cluster of Excellence "Integrative Production Technology for HighWage Countries".

\section{References}

1. Möller, N.: Bestimmung der Wirtschaftlichkeit wandlungsfähiger Produktionssysteme. Forschungsbericht IWB, Band 212 (2008)

2. Müller, R., Buchner, T., Fayzullin, K., Gottschalk, S., Herfs, W., Hilchner, R., Pyschny, N.: Montagetechnik und -organisation. Apprimus Verlag, Aachen (2009)

3. Lotter, B.: Montage in der industriellen Produktion. Ein Handbuch für die Praxis. Springer, Berlin (2006)

4. Nyhuis, P.: Wandlungsfähige Produktionssysteme. Heute die Industrie von morgen gestalten Garbsen, PZH, Produktionstechn. Zentrum (2008)

5. Licha, A.: Flexible Montageautomatisierung zur Komplettmontage flächenhafter Produktstrukturen durch kooperierende Industrieroboter. Meisenbach, Bamberg (2003)

6. Feldmann, K., Ziegler, C., Michl, M.: Bewegungssteuerung für kooperierende Industrieroboter in der Montageautomatisierung. wt werkstattstechnik online. Jg. 97(9), S.713 (2007)

7. Rückel, V., Feldmann, K.: Komplettmontage mit kooperierenden Robotern. wt werkstattstechnik online Jg. 95(3), S. 85 (2005)

8. Riedel, M.: Systematische Entwicklung und Konstruktion einer Handhabungseinheit zum Greifen und Manipulieren von Objekten auf Basis einer parallelkinematischen Struktur. Institut für Getriebetechnik und Maschinendynamik an der RWTH Aachen (2005)

9. Riedel, M., Nefzi, M., Corves, B.: Development and Design of a multi-fingered Gripper for Dexterous Manipulation. In: Mechatronic 2006, 4th IFAC-Symposium on Mechatronic Systems. in Eng., Heidelberg, September 12-14 (2006)

10. DE 102005059 349.6, Parallelkinematisches Greifsystem zum Greifen und Handhaben, PARAGRIP (2005)

11. Riedel, M., Nefzi, M., Hüsing, M., Corves, B.: An Adjustable Gripper as a Reconfigurable Robot with a Parallel Structure. In: Second International Workshop on Fundamental Issues and Future Research Directions for Parallel Mechanisms and Manipulators, Montpellier, France, September 21-22 (2008)

12. Müller, R., Corves, B., Hüsing, M., Esser, M., Riedel, M., Vette, M.: Rekonfigurierbares selbstoptimierendes Bauteilhandling. In: 8. Kolloquium Getriebetechnik Aachen 2009, Verlagshaus Mainz, Aachen, pp. S.297-S.311 (2009) ISBN: 3-86130-984-X 